\title{
Féeries
}

Études sur le conte merveilleux, XVII ${ }^{\mathrm{e}} \mathrm{XIX} \mathrm{X}^{\mathrm{e}}$ siècle

\section{La clé et le puits : à propos du déchiffrement des contes et des fables}

The Key and the Well: about the Deciphering of Tales and Fables

\section{Aurélia Gaillard}

\section{(2) OpenEdition}

1 Journals

\section{Édition électronique}

URL : http://journals.openedition.org/feeries/767

DOI : $10.4000 /$ feeries.767

ISSN : 1957-7753

Éditeur

UGA Éditions/Université Grenoble Alpes

\section{Édition imprimée}

Date de publication : 31 octobre 2010

Pagination : 179-192

ISBN : 978-2-84310-182-3

ISSN : 1766-2842

\section{Référence électronique}

Aurélia Gaillard, "La clé et le puits : à propos du déchiffrement des contes et des fables », Féeries [En ligne], 7| 2010, mis en ligne le 31 juillet 2011, consulté le 07 septembre 2020. URL : http:// journals.openedition.org/feeries/767 ; DOI : https://doi.org/10.4000/feeries.767

\section{(c) Féeries}




\section{Aurélia Gaillard}

Université Michel de Montaigne-Bordeaux 3, TELEM EA 4I 95

\section{LA CLÉ ET LE PUITS : À PROPOS DU DÉCHIFFREMENT DES CONTES ET DES FABLES}

«Il essaya la clé, elle allait parfaitement. Puis il la tourna une fois dans la serrure, et maintenant il nous faut attendre qu'il ait fini d'ouvrir et soulevé le couvercle, nous saurons alors quelles choses merveilleuses étaient contenues dans la cassette."

Grimm, La Clé d'or'.

"Ulysse, votre père, est le plus sage de tous les hommes; son cœur est comme un puits profond : on ne sauroit y puiser son secret."

FÉnelon, Télémaque, livre I8².

«Le beau, le bon, à mon gré, n'est que dans le vrai, mais où est ce vrai? Dans le fonds d'un puits.»

Le Téléscope ${ }^{3}$.

«Un Astrologue un jour se laissa choir $\mathrm{Au}$ fond d'un puits. On lui dit : Pauvre bête, Tandis qu’à peine à tes pieds tu peux voir, Penses-tu lire au-dessus de ta tête?»

La Fontaine, L'Astrologue qui se laisse tomber dans un puits ${ }^{4}$.

I. Grimm, Contes, Paris, Gallimard, coll. «Folio classique», 1976, p. 384-385.

2. Fénelon, Les Aventures de Télémaque, Paris, Classiques Garnier, 2009, p. 566.

3. Anonyme, Le Téléscope, petit conte moral, Moscou, I759, p. 7-8. Je remercie Magali Fourgnaud pour m’avoir fait découvrir ce conte.

4. La Fontaine, Fables (II, I3), dans Euvres complètes, Paris, Gallimard, coll. «Bibliothèque de la Pléiade", I99I, t. I, p. 87. 
$P$ LUS DE QUINZE ANS ont passé depuis que j'ai publiés Fables, mythes, contes - l'esthétique de la fable et du fabuleux (I660-I724), ouvrage issu de ma thèse : ce n'est pas un constat nostalgique mais je ne peux pas ignorer, ni reprendre non plus du même au même, ce qui avait été ma conviction, mon hypothèse, ma démonstration. Comme l'est l'écriture d'une version nouvelle d'un conte usé pour le conteur ou le fabuliste, écrire sur la question du conte et de la fable (de la Fable?) est aussi pour moi une sorte de gageure. Je ne ferai pas durer plus qu'il ne faut le nécessaire préliminaire; de ma thèse, je retiendrai néanmoins deux traits :

I. La démonstration générale reposait sur l'idée d'un "pacte allégorique» qui liait jusqu'autour de I670-I680 (le Traité du poème épique de Le Bossu, I675, la Demonstratio Evangelica de P.-D. Huet, I680) les différentes catégories de récits fabuleux (ce que nous appelons désormais la fable-apologue, le conte et le mythe) et imposait une lecture chrétienne, ou du moins la possibilité, l'attente, l'horizon d'une telle lecture. La Fable/le fabuleux était donc d'abord l'histoire d'un rapport au sens, rapport qui déterminait un champ commun entre toutes les formes de récits fabuleux, petite ou grande fable, conte. Ce pacte, une fois défait ou délié, les modalités d'écriture des récits fabuleux, notamment l'apologue et le conte, s'autonomisaient et des poétiques génériques se mettaient en place.

2. J'essayais ensuite d'établir une différenciation entre les trois modalités d'écriture du mythe, du conte et de la fable, qu’on peut résumer par une distinction entre écriture nostalgique pour le mythe (récit sacré), détachée pour le conte (dégradation du sacré) et ironique pour la fable (désacralisation). C'est cette nomenclature qui, désormais, m'apparaît, non pas vraiment sommaire ni inexacte, mais insuffisante, partielle.

Pour deux raisons : la première est que le lien entre la démonstration historique et l'enjeu poétique est peu explicité. Ainsi, à la relecture, j'ai tendance à me demander à moi-même : est-ce que ceci vaut pour toute fable, tout mythe, tout conte, de tous temps et de tous lieux? Mais alors, comment ceci (la faillite du pacte allégorique) a-t-il engendré cela (une vision éclatée et étagée des récits fabuleux)? La deuxième raison est que, dans l'ouvrage, j'appelle "temporalité» ou "temps», ce qui relève en fait d'un rapport au temps, du regard, de la reconstruction d'un temps absent/

5. Paris, Champion, 1996. 
passé : les modalités d'écriture du récit fabuleux à la fin du XVII ${ }^{\mathrm{e}}$ et au $\mathrm{XVIII}^{\mathrm{e}}$ siècles sont de fait étroitement liées à la question de la "survivance» des dieux antiques (et modernes) et à la construction d'une identité mémorielle. Jean-Paul Sermain a, depuis, très nettement montré combien la fondation du conte de fées classique et des Lumières procédait par recyclage et transposition d'un matériau ancien, dans une attitude "postcritique ${ }^{6}$ " et quelle était la proximité entre le conteur et, à la même époque, le personnage d'épistolier et de mémorialiste : "Ce qui rapproche le conte des écritures romanesques contemporaines est qu'il se présente comme une version écrite d'un antécédent oral sur lequel il attire le regard ${ }^{7}$.»

Aujourd'hui, lorsque je me penche à nouveau sur les ressemblances et différences entre la Grande Fable, la petite et le conte, je m'interroge : de quoi est-il question, au fond, dans les variations entre fable (apologue), mythe (histoire fabuleuse) et conte? D’herméneutique? De mémoire? Je souhaite saisir ici l'occasion de prolonger mes réflexions en reprenant d'une part la question de l'articulation historique et poétique et d'autre part en essayant de mieux préciser les modèles d'accès au sens des trois sortes de récits fabuleux, en partant de l'image du puits.

\section{La triple liquidation du fabuleux}

Ce qui opacifie les contours des trois catégories, c'est précisément ce qui aurait pu/dû les éclairer : le discours de légitimation du conte, de l'apologue ou de la «narration fabuleuse" pour reprendre l'expression célèbre de Fénelon à propos du Télémaque ${ }^{8}$. Il apparaît en effet que l'ambiguïté et la porosité entre ces trois sortes de récits fabuleux qui auraient dû, logiquement, disparaître avec la faillite du pacte allégorique et du champ unifié (toute proportion gardée) de la Fable, proviennent précisément et paradoxalement du moment et du geste qui ont déterminé leur refondation : la triple liquidation du fabuleux par Fontenelle, Bayle mais aussi Desmarets de Saint-Sorlin, Perrault, Terrasson, La Motte, comme mensonge (ou dissimulation), sottise (ou bagatelle ou extravagance) et immoralité (ou défaut de bienséance) ${ }^{9}$, dans la Querelle (des Anciens et des Modernes et plus

6. J.-P. Sermain, Le Conte de fées du classicisme aux Lumières, Paris, Desjonquères, 2005, notamment p. 55 .

7. Ibid., p. 92.

8. Fénelon, Lettre au Père Le Tellier, I710, dans Euvres de Fénelon, I848-1852, t. VII, p. 665-666.

9. Voir mon ouvrage, Fables, mythes, contes, ouvr. cité, part. I, chap. 2 ( La critique de la Fable et du fabuleux»), notamment p. IO2-I62; ainsi que l'ouvrage un peu ancien mais toujours éclairant 
précisément dans celle d'Homère) constitue désormais le socle à partir duquel peuvent se penser de nouvelles conditions de possibilité pour le récit fabuleux. Or ce socle amène bien une reconfiguration des rapports entre grande Fable, petite et conte mais toujours dans un rapport (renouvelé néanmoins) aux autres "fables" (au sens de récits fabuleux). Le conte s'édifie sur la perte de légitimité de la Fable grecque, tandis que celle-ci, en retour, se relégitime en tant que réservoir de contes émerveillants, quant à la petite fable (l'apologue), une fois le massif lafontainien contourné (provisoirement), elle semble bien ${ }^{\text {10 }}$ conserver, seule, le but didactique, anciennement commun aux trois - du moins maintient-elle ou tend-elle à maintenir un sens figuré réalisé textuellement ${ }^{\mathrm{II}}$.

Quelques exemples pour appuyer ces assertions peut-être un peu abruptes.

Perrault, d'abord, dont on connaît l'argumentation tissée depuis le Parallèle des Anciens et des Modernes jusqu'à la préface des Contes en vers de I695: pour lui, l'Iliade et l'Odyssée ne sont qu'un «tissu agréable d'aventures de héros, moitié vraies et moitié fabuleuses, composées pour plaire et pour instruire tout ensemble ${ }^{12}$ », tandis que les "contes faits à plaisir» peuvent, certes, apparaître comme "frivoles et bizarres ${ }^{13}$ ", mais ne sont pas pour autant «de pures bagatelles ${ }^{\mathrm{I} 4}$ ». À la triple «liquidation» de la Fable, à laquelle il contribue, Perrault répond alors par une triple légitimation du conte : premièrement, le conte n'est pas si frivole qu'il y parait, il est même moins extravagant ou incompréhensible que les contes des Anciens (les fables milésiennes, Psyché); deuxièmement, le conte respecte la bienséance ${ }^{15}$; enfin, le conte ne ment pas : il ne prétend pas à la philosophie ni à l'allégorie spirituelle (comme Psyché), mais à une empathie

de B. Magne, Crise de la littérature française sous Louis XIV : humanisme et nationalisme, Paris, Champion, 1976, 2 vol., notamment le développement sur «La fable et la raison» et «Mythologie et bienséance", p. 580-598.

Io. Voir l'étude qu'y consacre dans ce volume J.-N. Pascal, «Un couple fantomatique : le conte et la fable chez quelques fabulistes des Lumières".

II. C'est ainsi que J.-M. Schaeffer caractérise la fable, l'opposant à d'autres types de récits où la "transposition figurale» est à la charge du lecteur, comme par exemple La Divine Comédie (Qu'est-ce qu’un genre littéraire?, Paris, Seuil, coll. «Poétique», I989, p. III).

I2. Ch. Perrault, Parallèle des Anciens et des Modernes (I688-1697), t. I, «En ce qui regarde les arts et les sciences", dans J. Boch (éd.), L'Âge d'or du conte de fées : de la comédie à la critique, Paris, Champion, coll. "Bibliothèque des Génies et des Fées", n 5 , 2007, p. 357.

13. Ch. Perrault, Préface des Contes en vers, ibid., p. 364.

I4. Ibid., p. 36I.

15. "Tout ce qu'on peut dire, c'est que cette fable de même que la plupart de celles qui nous restent des anciens n'ont été faites que pour plaire sans égard aux bonnes mœurs qu'ils négligeaient beaucoup. Il n'en est pas de même des contes que nos aïeux ont inventés pour leurs enfants [...] le 
simple avec les bons et les méchants. Ainsi, les histoires «excitent dans les enfants le désir de ressembler à ceux qu'ils voient devenir heureux, et en même temps la crainte des malheurs où les méchants sont tombés par leur méchancetét ${ }^{16} »$. Surtout, quelle que soit l'image fantasmée du conte qu'est en train de construire Perrault, naïve, orale et populaire, enfantine, il s'agit de restituer un ensemble de qualités qui tout en n'étant pas de l'ordre de la superstition ou du préjugé, prennent ancrage dans l'irrationnel : une fable débarrassée des «erreurs de l'esprit humain ${ }^{17}$ ", pour reprendre le mot de Fontenelle, une fable sans la Fable, un «merveilleux vrai» débarrassé $\mathrm{du}$ fabuleux ${ }^{18}$. Pour ce dernier, en effet, la distinction entre «Fable» et "conte» est sans nul doute liée à la croyance/crédulité : la Fable, c'est, paradoxalement, quand l'histoire ne passe pas pour être fabuleuse, quand on y croit.

[...] Ces ornemens étoient faux, peut-être même que quelquefois on les donnoit pour tels; et cependant les histoires ne passoient pas pour être fabuleuses ${ }^{19}$.

Néanmoins, cette «croyance» n'est pas exactement semblable à celle supposée par les faits historiques ou les realia. C'est une sorte de croyance/ non croyance, de croyance contextualisée : on n'y croit pas pour aujourd'hui mais pour autrefois : "Y avons-nous la même foi qu'aux faits? Non ${ }^{20}$.»

On savoit que cela pouvoit n'être pas vrai; mais en ce temps-là il étoit vraisemblable, et c'en étoit assez pour conserver à ces Fables la qualité d'histoires ${ }^{21}$.

Au contraire, le conte (même si le mot n'est pas prononcé par Fontenelle dans ce discours dont ce n'est pas le propos) serait une histoire merveilleuse revendiquée comme telle. Les Métamorphoses d'Ovide ou les fables de Tchouang-tseu ${ }^{22}$ ne sont pas ainsi d'une autre qualité que les contes

désir de plaire ne m’a jamais assez tenté pour violer une loi que je me suis imposée de ne rien écrire qui pût blesser ou la pudeur ou la bienséance.» (Ibid., p. 363-365.)

16. Ibid., p. 92.

17. B. le Bovier de Fontenelle, De l'origine des fables, Paris, Fayard, 1989, CEuvres complètes, t. 3, p. 202.

I8. Voir l'étude que J. Boch consacre au «merveilleux sans la fable» dans ce volume.

19. De l'origine des fables, ouvr. cité, p. 193.

20. Ibid.

2I. Ibid., p. I94.

22. Ce sont les deux corpus donnés en exemple par Fontenelle (ibid., p. 198). Tchouang-tseu n'est pas identifié, Fontenelle ayant sûrement connaissance des contes philosophiques chinois par les jésuites, la China illustrata de Kircher (1667), ou par B. d'Herbelot dans la Bibliothèque orientale (1697). 
faits à plaisir («les histoires de faits imaginés à plaisir $\left.{ }^{23} »\right)$; seul le lien à la croyance est différent.

Quoique nous soyions incomparablement plus éclairés que ceux dont l'esprit grossier inventa de bonne foi les Fables si agréables pour eux; ils s'en repaissoient parce qu'ils y croyoient, et nous nous en repaissons avec autant de plaisir sans y croire ${ }^{24}$.

Le conte : la fable sans le mensonge, le plaisir du récit pur, sans l'illusion de vérité. C'est de cette définition négative, corollaire d'une délégitimation du fabuleux, que provient alors la nouvelle légitimité de la Fable $^{25}$, revalorisée de plus en plus sous l'appellatif de «mythologie» et non plus "d'histoire poétique». C'est la position du fameux "Chompré», le Dictionnaire abrégé de la Fable (I727), dont l'Avertissement souligne d'abord, dans la lignée de Fontenelle et de Bayle, le discrédit où est tombée la Fable :

On sait que la Mythologie est un tissu d'imaginations bizarres, un amas confus de faits $[\ldots]$ : qu'enfin c'est un assemblage de contes misérables, la plupart destitués de vraisemblance, et dignes de mépris ${ }^{26}$.

À quoi sert alors un tel dictionnaire? On sait qu’à la différence des anciennes "histoires poétiques», il ne sert plus à dévoiler, à donner le sens secret, le chiffre. "Nous n'avons pas entrepris d'expliquer ce qui est caché sous ces voiles ${ }^{27}$.» À quoi, alors? À comprendre les auteurs anciens ("pour l'intelligence des Poëtes»), à déchiffrer les tableaux, dit Chompré. Mais à quoi bon? C'est François-Xavier Rigord, une décennie plus tard (1738), qui franchit le pas: ce qui s'appelle désormais bien la «mythologie» sert, certes, à comprendre les auteurs anciens, mais plus encore à accéder aux «beautés» de ceux-ci.

Indépendamment des réflexions utiles que doit faire naître dans notre esprit la connoissance de la Mythologie, on peut dire qu'elle renferme de grands avantages, en ce qu'elle répand une grande lumière sur les Ouvrages des Anciens [...]. Cette connoissance en est la véritable clef, et sans elle une infinité de beautés nous échapperoient ${ }^{28}$.

23. De l'origine des fables, ouvr. cité, p. I94.

24. Ibid., p. 199-200.

25. Voir J. Boch, Les Dieux désenchantés. La fable dans la pensée française de Huet à Voltaire (I680I760), Paris, Champion, 2002.

26. P. Chompré, Dictionnaire abrégé de la Fable, Paris, Saillant \& Nyon, et Veuve Desaint, I777, I2 $2^{\mathrm{e}}$ éd., p. 3.

27. Ibid., p. 4.

28. F.-X. Rigord, Connoissance de la Mythologie, Paris, Étienne-François Savoye, 1753, 4 éd., p. IV-V. 
Et de fait, après un départ tout fontenellien ${ }^{29}$, l'ouvrage de Rigord, fait d'une certaine façon dériver la fable vers le conte enchanté : le dispositif en dialogue ("par demandes et par réponses») qu'il adopte n'est pas seulement celui d'un pédagogue, il contribue aussi à faire des récits fabuleux autant de contes rapportés, le mythologue-pédagogue devenant conteur, c'est-à-dire avant tout rapporteur, passeur. À la question de la vérité de la Fable, que Fontenelle a tournée en questionnement sur l'origine de l'affabulation, Rigord, tout en conservant une vision religieuse, répond par la pérennité d'une transmission.

Par exemple : à la question «L'aventure d'Énée et de Didon a-t-elle quelque chose de vrai?» On trouve comme réponse : «Rien n'est plus fabuleux et plus contraire à l'Histoire ${ }^{30}$. " Même chose pour Énée seul : "M. Samuel Bochart a prouvé qu'Énée n'étoit jamais venu en Italie ${ }^{3 \mathrm{I}}$. Mais suit alors ce qui se présente comme des contes "rapportés»: "Cependant l'opinion la plus reçue est qu'il y a eu un Énée fils d'Anchise, qu'il épousa Créüse $[\ldots]^{32}$.» La plupart du temps, les «demandes» mêmes intègrent cette donnée : "Que rapporte-t-on des Alcyons?33» ou "Que raconte-t-on de Diomède? ${ }^{34}$ ».

Or, c'est précisément cet «encadrement» de l'histoire fabuleuse racontée qui contribue encore à transformer la Fable en conte et l'Histoire poétique en recueil de contes ${ }^{35}$. À partir de là, on comprend mieux comment l'ouvrage apparemment obsolète de Le Bossu, avec son assimilation totale de la grande et de la petite fable, le Traité du poème épique de 1675, est sans cesse réédité, en I693, I7I4, et pendant toute la première moitié du $\mathrm{XVIII}^{\mathrm{e}}$ siècle alors même que son "contenu» pouvait sembler périmé après la Querelle d'Homère. En effet : son point de départ et la clé de voûte de toute sa démonstration est une définition commune à toutes les catégories du fabuleux (apologue, récit épique/mythe, conte) : «La Fable est un discours inventé avec art pour former les mœurs par des instructions

29. «Il suffira de faire remarquer à ce sujet, que quoique nous soyons désabusés des erreurs du Paganisme, la connoissance de la Mythologie, qui n'est autre chose que l'histoire des erreurs où les Payens ont été si long-tems ensevelis, nous doit faire sentir le bonheur que nous avons d'être guidés par la vérité [...].» (Ibid., p. Iv.)

3o. Ibid., p. 393.

31. Ibid., p. 398.

32. Ibid.

33. Ibid., p. 146.

34. Ibid., p. 373 .

35. Sur l'importance du «recueillement» comme geste mémoriel, voir le $\mathrm{n}^{\circ} \mathrm{I}$ de Féeries, «Le Recueil», Grenoble, Ellug, 2003. 
déguisées sous les allégories d'une action ${ }^{36}$.» Toute fable était donc, pour Le Bossu et son époque ${ }^{37}$, par essence allégorique, c'était un récit voilé, avec un but didactique. Une telle définition avait d'abord une fonction légitimante pour la Fable païenne, aussi était-il essentiel que soient gommées au maximum les différences entre Grande et petite fable :

Concluons donc, que le nom de Fable donné à la Fable de l'Iliade, et à celle d'Ésope, n'est ni équivoque ni analogue, mais synonyme et également propre; que toutes les conditions qui y mettent quelques différences, ne touchent aucunement le fond, la nature ni l'essence de la Fable, mais qu'elles n'en sont que diverses espèces ${ }^{38}$.

Quel usage peut donc encore avoir l'ouvrage de Le Bossu après la Querelle - en dehors d'un usage partisan anti-moderne ici ou là? Thémiseul de Saint-Hyacinthe, polygraphe qui a stigmatisé le « respect aveugle de l'Antiquitée ${ }^{39}$ " dans Le Chef-d'œuvre d'un inconnu (I7I4) ${ }^{40}$, dit et justifie son admiration pour le R. Père dans une préface à l'édition hollandaise qu'il lui consacre et, par là, nous éclaire sur cette réception inattendue :

Les choses que ces grands Hommes [Homère et Virgile] ont dites, et qui paroissent les plus absurdes à ceux qui les lisent sans connoître le génie de la Poësie Épique, deviennent des choses admirables pour ceux qui savent en juger. On est ravi de se trouver l'esprit entretenu de vérités instructives, lorsque l'imagination est agréablement occupée par des fictions merveilleuses ${ }^{41}$.

Saint-Hyacinthe rejoint ainsi, à sa façon, Rigord : la Fable est devenue, pour partie au moins, une "fiction merveilleuse», un conte qui, agréablement berce. Fontenelle d'ailleurs (toujours) avait déjà suggéré cette lecture dans ses Nouveaux dialogues des morts (I683) et son parallèle moqueur entre Ésope et Homère (Dialogues des morts anciens, V) : il ne s'agissait plus alors d'établir de savantes correspondances allégoriques ou «morales» entre les deux sortes de fables, mais simplement d'en montrer la proximité du point de vue de l'invention, semblablement ingénieuse, surprenante, agréable. Les deux sortes de «fables» devenaient ainsi de simples

36. R. Le Bossu, Traité du Poëme épique, Paris, André Pralard, I693 [I675], p. 9-IO.

37. Je renvoie à la première partie de mon ouvrage, Fables, mythes, contes, ouvr. cité.

38. R. Le Bossu, ouvr. cité, p. 37.

39. Selon le mot de Fontenelle dans De l'origine des fables, ouvr. cité, p. 196.

40. Rééd. par H. Duranton, Publications de l'Université de Saint-Étienne, I99I.

4I. Préface de Thémiseul de Saint-Hyacinthe à la $6^{e}$ édition du Traité du poème épique, augmentée de remarques, d'un discours préliminaire sur l'excellence de l'ouvrage, de R. Le Bossu, La Haye, H. Scheurleer, I7I4, p. viII. 
contes : l'apologue est explicitement appelé un "petit conte ${ }^{42} »$, la narration fabuleuse, par assimilation, devenant alors un «grand» conte. Grande et petite fable sont devenus grand et petit conte. Le glissement sémantique témoigne alors d'une transformation idéologique et anthropologique. L'adossement de la narration fabuleuse n'est plus à une transcendance mais à une mémoire.

Et à l'inverse : le conte se légitime en usant des arguments pourtant désuets de la Fable tels qu'ils étaient notamment énoncés par Le Bossu. Revenons une nouvelle fois à Perrault :

Je prétends même que mes fables méritent mieux d'être racontées que la plupart des contes anciens, et particulièrement celui de la Matrone d'Éphèse et celui de Psyché, si l'on les regarde du côté de la morale, chose principale dans toute sorte de fables, et pour laquelle elles doivent avoir été faites ${ }^{43}$.

C'est revenir à la définition de Le Bossu : une "instruction déguisée», leçon qui doit d'ailleurs déterminer entièrement et premièrement la narration. Pour Le Bossu, la "fable» se construit à l'envers, en partant de la fin. Perrault se saisit donc de l'argumentation qui vise d'abord l'épopée/le mythe ou l'apologue et, tout en disqualifiant ceux-ci (La Matrone d'Éphèse, Psyché), il s'en sert pour légitimer le conte, nouvelle manière.

La triple liquidation du fabuleux s'est donc accompagnée d'une relégitimation complexe des différentes sortes de récits fabuleux par confrontation, emprunt, désaveu : la difficulté à appréhender les différences entre les catégories du fabuleux, pour le XviII ${ }^{\mathrm{e}}$ siècle, provient donc du glissement qui s'est opéré dans la conception et par là même dans la pratique du conte et des «fables» (grande et petite). Alors qu'avant I670, on pourrait dire que tout (ou presque) était fable, après I700, tout (ou presque) devient conte. "Contes pour contes", écrit Marie-Jeanne Lhéritier, en comparant la Fable grecque et les contes "gaulois», «les fées ne sont pas moins en droit de faire des prodiges, que les dieux de la fable ${ }^{44} »$. C'est là, sans doute, qu'intervient, exemplairement, La Fontaine : comme auteur de la bigarrure et de la porosité entre les catégories du conte, de l'apologue mais aussi de la "narration fabuleuse» (Les Amours de Psyché) ${ }^{45}$. Non

42. Fontenelle, Nouveaux dialogues des morts, dans Euvres diverses, Paris, Michel Brunet, I724, p. 20.

43. Ch. Perrault, Préface des Contes en vers, ouvr. cité, p. 362-363.

44. M.-J. Lhéritier de Villandon, Les Enchantements de l'éloquence, dans L'Âge d'or du conte de fées, ouvr. cité, p. 370.

45. Voir l'étude que P. Dandrey consacre, dans ce volume, à partir du Livre XII des Fables, à la question, sous le titre "Les fables, les contes et la Fable chez La Fontaine». 
seulement La Fontaine pratique les trois sortes de récits fabuleux, non seulement il théorise sa pratique mais encore et surtout il mêle conte, fable et "mythe", au sein des recueils, voire au sein d'une même pièce. D'une certaine façon, on peut dire qu'il pratique la fable en conteur et parfois le conte en fabuliste (par exemple "On ne s'avise jamais de tout» ou «Le villageois qui cherche son veau ${ }^{46}$ ). Mais, qu'entend-on alors par "fable» et "conte», ou même par Fable, fable et conte? Si tout était "fable» et que désormais tout est "conte», comment s'énoncent les nouvelles différences? Et surtout, qu'indiquent-elles sur les différences fondamentales entre les trois sortes de récits fabuleux? J'avancerai quelques hypothèses.

\section{La clé et le puits}

J'ai fait silence jusqu'à présent sur les quatre citations mises en exergue, prises à quatre genres, moments et univers apparemment distincts : un apologue lafontainien (I668), la «narration fabuleuse» de Fénelon (I699), un petit conte anonyme, Le Téléscope (1759), un conte populaire de Grimm (I8I2-I829). Il est temps d'y venir.

Les quatre textes assimilent la quête de sens du récit fabuleux à la quête d'un secret, enfoui. Le puits (ou la cassette de Grimm, ou le coffret de Freud ${ }^{47}$, ou le souterrain de Riquet, ou encore chez Fénelon, dans L'Anneau de Gygès, l'urne dans la caverne) en est l'image exemplaire : tout conte ou mythe postule l'existence d'un puits, toute lecture est descente, catabase. Mais dans le même temps, même geste, conformément à la nature profonde du secret qui est de ne pas être dévoilé, faute de quoi, il s'évanouit, la vérité, le trésor, ne doivent pas être trouvés dans le puits, la clé qui semble ouvrir la serrure est toujours un leurre — soit qu' elle ouvre sur l'horreur (La Barbe bleue), soit qu'elle délivre un pseudo-trésor (l'anneau de Gygès). Soit encore que, faisant l'économie de la clé (alors que tout le trésor est précisément dans la clé et non dans ce qu'elle ouvre, comme tout le secret est dans le secret $\left.{ }^{48}\right)$, le puits lui-même s'offre comme

46. La Fontaine, Contes et nouvelles en vers, dans $O C$, ouvr. cité, p. 659 et p. 66I.

47. S. Freud, "Le thème des trois coffrets" (1913), dans Essais de psychanalyse appliquée, Paris, Gallimard, coll. «Idées», I97I, p. 87-IO3.

48. "Ce qui revient à dire que le secret paraît bien ne pas être, ne pas pouvoir être une chose, un être, un mot, une pensée, un discours, un "ce-qui" mis à l'écart; seulement une apparence ou une apparition, une enveloppe légère, incorporelle, qui flotte sur les choses [...]. Le secret, un incorporel, un simulacre, un rien." (L. Marin, "Logiques du secret», dans Lectures traversières, Paris, Albin Michel, 1992, p. 248.) 
leurre : et c'est alors la chute, de l'Astrologue ou du bouc (Le Renard et le Bouc, III, 5).

C'est le conteur de Grimm qui nous renseigne le mieux sur ce que sont le conte et le mythe, le conte à portée mythique : l'histoire de cette "clé d'or" est clairement celle du conte, du conteur, du contage et du lecteur-auditeur. C'est l'histoire d'un "pauvre gars», en plein hiver, "un jour que la neige était épaisse» (et cela compte/conte bien sûr, puisqu’il va falloir écarter, soulever toute cette neige) qui trouve une "petite clé d'or»: la même que celle du cabinet de Barbe-bleue, la clé fée des contes, également clé des contes, outil de déchiffrement. Et c'est là que le conteur se moque (gentiment) du lecteur : "Il crut alors que là où était la clé, la serrure devait y être aussi ${ }^{49}$.» Or le conte enseigne précisément que «là où est la clé», il n'y a pas de serrure : soit qu'il n'y en ait pas du tout, soit que celle-ci justement soit disposée ailleurs dans le conte. Mais le plus retors dans ce conte est que l'effet est redoublé : car le pauvre gars finit bien par trouver une serrure, celle d'un coffret de fer (fer du coffret contre or de la clé n'augure rien de bon). Et la moquerie du conteur consiste alors à laisser en attente la découverte, à ralentir le mouvement de l'ouverture et à en suspendre geste et sens. Le déchiffrement (la clé du conte) est bien dans le mouvement de sa lecture et non dans sa solution/dissolution. C'est bien un «puits profond» et inépuisable. L'image du puits et/ou de la clé renseigne donc sur la nature comparée des déchiffrements entre conte, mythe et fable: si l'essence de tout récit fabuleux réside dans l'épaisseur d'un sens à déchiffrer, la différence entre les trois types de récits fabuleux est bien au moins autant dans la manière dont ce sens est à déchiffrer que dans la nature du sens (sacré, désacralisé, parodique).

Ainsi la grande Fable et le conte partagent-ils un même mode de déchiffrement : ils signalent l'existence d'un sens caché, d'un "puits» installé quelque part, c'est toujours l'histoire d'un trésor que l'on cache, d'un secret (l'histoire d'Eedipe et de son secret ${ }^{5 \circ}$ ); mais ce sens caché est, dans le même temps, mis sous clé, fermé à double tour : le lecteur est convié non pas à le déchiffrer mais à en écouter la transmission, la répétition, la réverbération d'un récit à l'autre, d'une version à une autre, d'une époque à une autre. Ce n'est pas tant un récit à clés qu'un recueil, qu'un rapport. Une question de mémoire plus que d'herméneutique. Tous deux sont toujours des «contes usés»:

49. Grimm, «La Clé d'or», dans Nouveaux contes, ouvr. cité, p. 226.

50. Voir L. Marin, «Logiques du secret», ouvr. cité, p. 247-257. 
S’il est un conte usé, commun, et rebattu,

C'est celui qu'en ces vers j'accommode à ma guise ${ }^{5 \mathrm{I}}$.

Pour Perrault, c'est d'ailleurs l'indice de cette voix «rapporteuse» et la figuration d'une véritable scène de contage qui permet d'assimiler Fable et conte :

Aussi voyons-nous qu'Apulée le [récit de Psyché] fait raconter par une vieille femme à une jeune fille que des voleurs avaient enlevée, de même que celui de Peau d'Âne est conté tous les jours à des enfants par leurs gouvernantes, et par leurs grands-mères ${ }^{52}$.

Pareillement, le conte, chez La Fontaine (même s'il ne s'agit pas de conte de fées) s'affiche d'abord comme une histoire de transmission, de reprise ("conte tiré de») et comme un contage :

Un paysan son seigneur offensa.

L'histoire dit que c'était bagatelles3.

Et c'est même à cette occasion, lors de l'exhibition insistante d'une généalogie de conteurs dans laquelle il s'agit de trouver place, que ressurgit une nouvelle fois l'image du puits :

Boccace n'est le seul qui me fournit.

Je vas parfois en une autre boutique.

Il est bien vrai que ce divin esprit

Plus que pas un me donne de pratique.

Mais comme il faut manger de plus d'un pain

Je puise encore en un vieux magasin;

Vieux, des plus vieux, où Nouvelles Nouvelles

Sont jusqu'à cent, bien déduites et belles

Pour la plupart, et de très bonne main ${ }^{54}$.

Dans ce puits, point de secret, mais, au contraire, une infinité d'autres histoires : le puits est un puits sans fond dans lequel s'entassent les contes ou, en abyme, des histoires de puits... Quant à la différence entre conte et mythe (ou grande Fable), même si la fin du Classicisme et les Lumières tendent à l'ignorer, elle serait alors plutôt de degré : la Fable serait un conte sacré, tendu vers la fondation du monde, le conte, un récit humain, laïc, tendu vers la refondation d'un ordre humain 5 . Comme témoignage

51. La Fontaine, La Matrone d'Éphèse (XII, 26), dans Fables, ouvr. cité, p. 508.

52. Ch. Perrault, Préface des contes en vers, dans L'Âge d'or du conte de fées, ouvr. cité, p. 362.

53. La Fontaine, Conte d'un paysan qui avait offensé son seigneur, dans OC, ouvr. cité, p. 590.

54. La Fontaine, La Servante justifiée, dans OC, ouvr. cité, p. 637. Je souligne.

55. Voir dans ce volume la seconde contribution de J.-P. Sermain, «Ce que les contes doivent aux fées". 
du double mouvement anthropologique du conte et de la Fable ${ }^{56}$, on pourrait alors penser à ces textes qui prétendent donner «l'explication des fables" (Rigord ou Banier ${ }^{57}$ ) ou «l'origine des fées" $\left(\right.$ Durand $\left.^{58}\right)$. Chercher l'origine de l'un ou l'autre de ces récits fabuleux, c'est simplement revendiquer une origine, les inscrire dans un mouvement anthropologique, dans une mémoire collective, une histoire fondatrice. Même chose pour le conte étiologique (L'Inconstance punie ou l'Origine des cornes, L'Origine du Lansquenet de $\mathrm{M}^{\mathrm{me}} \mathrm{d}^{\prime}$ Auneuil).

Et la petite fable? Qu'on pense au puits dans lequel l'Astrologue se prend les pieds ou à celui dans lequel se retrouvent Renard et Bouc assoiffés. Ce sont bien des puits parodiques : dans L'Astrologue 59 , le puits est l'outil d'un renversement du haut (les cieux, le front dans les étoiles) en bas (le fond d'un puits), dans Le Renard et le Bouc, le «secret" n'est plus au fond du puits, mais dans la manière d'en sortir. Le Bouc au Renard:

Par ma barbe, dit l'autre, il est bon; et je loue

Les gens bien sensés comme toi.

Je n’aurais jamais, quant à moi,

Trouvé ce secret, je l'avoue ${ }^{60}$.

L'apologue fait donc l'économie du puits — c'est-à-dire du mouvement anthropologique, de l'adossement à un sens supérieur, transcendance ou ordre humain. Mais, en tant que récit fabuleux, il partage avec le conte et la grande Fable, la double articulation du sens (littéral/figuré). Or, justement, ce qui le caractérise, c'est le jeu sur le double sens : dans la petite fable, les clés sont multiples et si possible contradictoires. C'est le règne du voile et de la gaze : rien n'est jamais dévoilé complètement ni rien n'est jamais caché totalement. C'est le mouvement oscillatoire du voilél dévoilé, du montré/caché qui détermine le déchiffrement. On pourrait lui appliquer l'exclamation du villageois de La Fontaine qui cherche son veau, dans le conte-apologue éponyme :

Ô dieux, que vois-je, et que ne vois-je pas! ${ }^{6 r}$

56. Sur le caractère mythique des contes, voir N. Belmont, "Contes populaires et mythes", dans le Dictionnaire des mythologies, Y. Bonnefoy (éd.), Paris, Flammarion, I98I, t. I, p. 209-213.

57. A. Banier, Explication historique des fables où l'on découvre leur origine et leur conformité avec l'Histoire ancienne, Paris, François Le Breton, $2^{\mathrm{e} e ́ d ~ a u g m e n t e ́ e ~ d ' u n ~} 3^{\mathrm{e}}$ vol., I7Is [I $\mathrm{I}^{\mathrm{re}}$ éd. I7II].

58. C. Bédacier, née Durand, Les Petits Soupers de l'été 1699, ou Aventures galantes avec l'origine des fées, Paris, Musier et Rolin, I702.

59. La Fontaine, L'Astrologue qui se laisse tomber dans un puits, ouvr. cité.

6o. La Fontaine, Le Renard et le Bouc (III, 5), dans OC, ouvr. cité, p. II4.

6r. La Fontaine, Le Villageois qui cherche son veau, dans $O C$, ouvr. cité, p. 66I. 
Quinze ans après Fables, mythes, contes ${ }^{62}$, je me rends compte combien les débats ont avancé, mais aussi combien la question ou les questions restent complexes et les réponses toujours insatisfaisantes : sans doute à cause de La Fontaine ou de Perrault qui «cachent» un peu la forêt et ont des positions et des pratiques originales et pas forcément représentatives. La Fontaine surtout qui, souvent, pratique la fable en conteur et le conte en fabuliste. Ce qui ressort des manifestes et poétiques comme des récits eux-mêmes, c'est bien, néanmoins, une proximité qui perdure, après la liquidation de la grande Fable grecque, entre fable, conte et mythe. Une proximité, mais aussi un déplacement : le couplage n'est plus le même, il n'est plus entre grande et petite Fable mais entre conte et grande Fable, grand Conte et petit conte. L'apologue est donc coupé d'une certaine façon du conte et de la Fable — sauf lorsqu'il se fait ou tend à se faire conte ou mythe (La Matrone d'Éphèse, Philémon et Baucis, ou Les Souhaits ${ }^{63}$ ). Néanmoins, les contes (Grand et petit) continuent à miroiter dans la fable, tout au fond du puits, comme des traces mémorielles : après tout, la leçon du Renard et le Bouc reste qu'il ne faut pas descendre "à la légère» dans un puits.

62. Ouvr. cité.

63. La Fontaine, Fables, dans OC, ouvr. cité, respectivement (XII, 25 et 26) et (VII, 5). 